\title{
Diffusion-weighted imaging-detected ischemic lesions associated with flow-diverting stents in intracranial aneurysms: safety, potential mechanisms, clinical outcome, and concerns
}

\author{
Christina losif, MD, MSc, PhD, ${ }^{1}$ Yann Camilleri, MD, ${ }^{2}$ Suzana Saleme, MD, ${ }^{1}$ François Caire, MD, ${ }^{3}$ \\ Catherine Yardin, MD, PhD, ${ }^{4}$ Sanita Ponomarjova, MD, ${ }^{1}$ Marie-Paule Boncoeur-Martel, MD, ${ }^{2}$ \\ and Charbel Mounayer, MD, PhD'
}

\begin{abstract}
Departments of ${ }^{1}$ Interventional Neuroradiology, ${ }^{2}$ Diagnostic Neuroradiology, and ${ }^{3}$ Neurosurgery, Dupuytren University Hospital; and ${ }^{4}$ Department of Histology, Cytology, Cellular Biology and Cytogenetics, Mother and Child (HME) University Hospital, Limoges, France
\end{abstract}

\begin{abstract}
OBJECT It was initially considered safe for flow-diverting stents to cover the ostia of branching vessels during endovascular procedures for the treatment of intracranial aneurysms. As more recent evidence suggests, however, their use is not always free of ischemic concerns in terms of covered arterial ostia. The authors sought to determine the frequency of silent and clinically evident diffusion-weighted imaging (DWI)-detected abnormalities related to stent placement as a means of elucidating potential clinical risks.
\end{abstract}

METHODS This is a prospective single-center study on a series of patients with intracranial aneurysms that were treated with flow-diverting stents. All patients systematically underwent an MRI protocol that included DWI before treatment, between 24 and 48 hours postprocedure, and 3 months postembolization. Effectiveness of anticoagulation treatment was assessed for all patients. Lesions seen on DWI were correlated to the parent artery and the side-branch territories and were statistically analyzed in relation to their time of occurrence and clinical presentation. The authors compared the DWI findings in these patients to findings obtained in patients treated with a stent-assisted coiling technique during the same time period.

RESULTS Over the course of 18 consecutive months, 38 consecutive patients ( 7 males and 31 females) with 49 intracranial aneurysms were treated using flow-diverting stents. Overall, $81.6 \%$ of the DWI spots found remained clinically silent during the follow-up period. Five ischemic clinical complications $(13.2 \%)$ occurred in the postprocedural period. No statistically significant correlation could be established between DWI spots or aneurysm location and clinical complications or follow-up modified Rankin Scale score at 3 months. The complication rate was $7.8 \%(n=3)$ at the 3-month follow-up, and mortality was $0 \%$. Compared with stent-assisted coiling, use of flow-diverting stents showed a statistically significant correlation with silent DWI findings postintervention.

CONCLUSIONS Lesions seen on DWI resulting from the procedure are far more common than anticipated, but the technique remains safe and effective, providing an interesting alternative for "difficult" aneurysms, regardless of location. Late-occurring DWI-detected lesions distal to side arterial branches imply a local pressure gradient drop, related to flow competition by collateral networks. Further research is needed to assess the extent and significance of these events. http://thejns.org/doi/abs/10.3171/2014.10.JNS132566

KEY WORDS intracranial aneurysms; brain ischemia; diffusion-weighted imaging; vascular disorders; stent

\footnotetext{
ABBREVIATIONS $\mathrm{ACA}=$ anterior cerebral artery; $\mathrm{AChA}=$ anterior choroidal artery; $\mathrm{ACOA}=$ anterior communicating artery; $\mathrm{ARU}=$ aspirin reaction unit; $\mathrm{clCA}=\mathrm{cavern}-$ ous segment of the internal carotid artery; DSA = digital subtraction angiography; $\mathrm{DWI}=$ diffusion-weighted imaging; $\mathrm{ECA}=$ external carotid artery; $\mathrm{MCA}=$ middle cerebral artery; $\mathrm{mRS}$ = modified Rankin Scale; OKM = O'Kelly and Marotta; PCoA = posterior communicating artery; PRU = P2Y12 reactivity unit; SAH = subarachnoid hemorrhage; $\mathrm{VA}=$ vertebral artery.

SUBMITTED November 22, 2013. ACCEPTED October 7, 2014

INCLUDE WHEN CITING Published online January 6, 2015; DOI: 10.3171/2014.10.JNS132566.

DISCLOSURE The authors report no conflict of interest concerning the materials or methods used in this study or the findings specified in this paper.
} 
$\mathrm{L}$ OW-POROSITY braided tubular endovascular devices, known as flow-diverting stents or flow diverters, offer a new therapeutic concept in the treatment of intracranial aneurysms and provide an interesting alternative to conventional coiling or assisted coiling techniques, especially for patients harboring aneurysms with anatomical configurations or characteristics that make conventional techniques inefficient (such as overly wide, large-necked saccular aneurysms or blister-like aneurysms). . $^{3,2,14,15,19}$

A flow diverter is a braided stent consisting of a thick, flexible, tubular mesh. Because of its mechanical properties, when a flow diverter is deployed at the level of an aneurysm neck, it causes stagnation of blood, thus promoting thrombosis of the aneurysm sac while providing scaffolding for endothelialization. Initially these devices were considered to provide an "anatomical cure" for intracranial aneurysms, but more recent evidence has raised concerns about the technique because of ischemic complications involving both the parent vessel and the side branches covered by the device. ${ }^{9}$

While mechanisms involved in ischemic complications have not yet been exhaustively studied and absolute indications for treatment with flow diverters are still debated, the incidence of hyperacute ischemic events related to flow diverter placement may provide an insight into this matter. While large prospective studies are mandatory, until then, the present study may provide useful points for further research and help in everyday decision making.

\section{Methods}

This prospective study comprises a single-center case series of patients with intracranial aneurysms who were treated with flow-diverting stents during a period of 18 months (January 1, 2012, to July 1, 2013). Patients with ruptured and unruptured intracranial aneurysms who underwent treatment with flow-diverting devices at our center, including those undergoing retreatment, were included. The choice of flow-diverting devices over regular or stent-assisted coiling was based on the usual indications, including recanalized aneurysms needing retreatment after previous embolization, large and giant aneurysms, and multiple or blood blister-like aneurysms, especially located in the same arterial branch. Single, regular-sized aneurysms were treated using flow-diverting stents in cases of aneurysms with a difficult-to-treat configuration related to dome-to-neck and aspect ratios. ${ }^{9}$

Patients provided written consent after they were made fully aware of the treatment's risks and benefits. In cases of unruptured aneurysm the patient's strong desire to be treated, expressed during consultation, was also considered during decision making.

\section{Patient Population and Aneurysm Description}

Consecutive patients who were treated for ruptured and unruptured intracranial aneurysms were considered for inclusion on the basis of the following criteria: one or more aneurysms treated with one or more flow diverters and age older than 18 years. Exclusion criteria for the present study were contraindication to MRI, treatment of another malformation or aneurysm with another technique at the same time, clopidogrel or aspirin resistance without normalization of the P2Y12 reactivity unit (PRU) or aspirin reaction unit (ARU) after dose adjustment, patient refusal, or inability to give consent. Demographic, clinical, and imaging data were registered in the department's prospectively maintained database and were quantitatively recorded in an appropriate electronic worksheet. Imaging data were reviewed and inserted accordingly, as described below.

Aneurysm location was categorized as anterior cerebral artery (ACA), anterior communicating artery (ACoA), middle cerebral artery (MCA), anterior choroidal artery (AChA), carotido-ophthalmic, posterior communicating artery (PCoA), cavernous segment of the internal carotid artery (cICA), carotid bifurcation at the superior end of the ICA where it divides into the ACA and MCA (also known as the carotid T junction), and vertebral artery (VA), based on the vessel harboring the aneurysm.

The technical complexity of the aneurysm in terms of anatomical morphology, a parameter that was largely considered in deciding to use a flow diverter rather than conventional endovascular techniques, was evaluated by calculating the dome-to-neck and aspect ratios ${ }^{9}$ for each aneurysm. Regarding aneurysms that were considered for retreatment, recanalization was classified according to the Raymond-Roy classification as complete occlusion, residual neck, or residual circulating aneurysm..$^{18}$

\section{Procedure-Related Medication}

All patients without a history of subarachnoid hemorrhage (SAH) were placed on a regimen of $75 \mathrm{mg}$ clopidogrel 6 days prior to the procedure, after being tested for proper response to clopidogrel with the Verify Now P2Y12 test (Accumetrics). An antiplatelet count higher than $100 \mathrm{~g} / \mathrm{L}$ and a hematocrit greater than $30 \%$ were ensured for patients before testing to acquire reliable results. If resistance occurred, the doses were substantially increased (tripled) and the patients were retested several days later. Patients with persistent abnormal values were excluded from this study. Aspirin resistance was also tested using the Verify Now Aspirin test (Accumetrics), and when resistance occurred, doubling of the follow-up doses was prescribed (taken 2 times per day).

During the procedure, intravenous heparin perfusion was administered, with the goal of maintaining an activated clotting time at 2 times the normal value. In addition, all patients were given $250 \mathrm{mg}$ aspirin intravenously. In the setting of SAH, an intravenous bolus of $0.25 \mathrm{mg} / \mathrm{kg}$ abciximab was administered during the procedure and a continuous intravenous perfusion of $0.125 \mu \mathrm{g} / \mathrm{kg} / \mathrm{min}$ for 12 hours was maintained immediately after the procedure.

After the procedure, patients were kept on a regimen of clopidogrel for 6 months and aspirin for 1 year. In cases in which there had been resistance to aspirin, the doses were increased and were given twice daily (morning and evening) by mouth.

\section{Treatment Procedure}

All procedures were performed by 2 experienced senior interventional neuroradiologists using a biplane flat panel digital subtraction angiography (DSA) unit (Allura 
Xper FD20, Philips). Uniformity of embolization was ascertained using a standardized imaging and technique protocol, which included the use of the same flow-diverting device (Pipeline Embolization Device, ev3 Inc.) and the same navigation and delivery material. For large and giant aneurysms, the adjunctive use of loose coil packing was considered in order to prevent delayed aneurysm rupture. The procedures were performed under general anesthesia, using coaxial endovascular navigation to achieve good support for flow diverter navigation: a long 6-F introducer (Destination, Terumo, or Neuron Max; Penumbra) was placed within the cervical portion of the ICA or VA; a 6-F guiding catheter (Navien, Covidien) and a Marksman delivery microcatheter were then advanced in a triaxial fashion.

Angiographic images were acquired in anteroposterior, lateral, and working projections before and after treatment. Rotational 3D angiograms with volumetric reconstruction were generated before stent positioning, with appropriate measures of the aneurysm(s) and the involved arterial parent branches to evaluate the diameter and length of the segment and to choose the appropriate dimensions of the flow-diverting stent(s). High-resolution CT scanning was performed at the end of treatment to evaluate flow diverter deployment.

Immediate angiographic outcome was categorized as complete occlusion, residual stasis, and residual aneurysm. The number of flow diverters deployed was decided on the basis of anatomy or flow reduction observed during the procedure.

\section{Clinical Evaluation Before and After Treatment}

Every patient underwent full clinical and neurological evaluation performed by a senior interventional neuroradiologist and a senior anesthesiologist before treatment, at awakening, at discharge, and at the 3-month followup. Modified Rankin Scale (mRS) scores were recorded. The clinical situation at awakening was classified as unchanged, deteriorated, delay in awakening, or absence of awakening. Before treatment and at follow-up, the cognitive state of the patients regarding orientation, language, calculation, memory, and visuospatial reproduction was evaluated. Quantification and documentation of the results were achieved using the Mini-Mental State Examination.

\section{Complications}

Complications occurring during the procedure were classified as technical challenges, aneurysm rupture, and inadvertent thrombus formation. Complications occurring after the procedure were categorized into procedure-related stroke, non-procedure-related stroke (defined as stroke occurring after the procedure, not present on the 24-hour DWI, and/or stroke developing in branches not related to the embolization), early or delayed rupture of the aneurysm, stent migration, and mass effect (perianeurysmal brain inflammatory reaction).

\section{Imaging Evaluation}

All MRI and DSA studies were reviewed independently by 2 senior neuroradiologists (C.I. and C.M.). If dis- agreement occurred, a decision was taken by consensus, after review of MRI and DSA studies.

Selective DSA of 4 axes (the 2 internal carotid and 2 vertebral arteries) was performed in every patient; in patients who harbored a carotido-ophthalmic aneurysm, selective external carotid artery (ECA) angiograms were also acquired. The same protocol was used at the 6-month angiographic follow-up.

The immediate angiographic outcome was categorized according to the Raymond-Roy classification as complete occlusion, residual stasis, and residual aneurysm. ${ }^{18}$ On follow-up DSA, we used the technique proposed by O'Kelly and Marotta ${ }^{16}$ (OKM grading scale) to evaluate the angiographic result, which was categorized as total filling of the aneurysm (Grade A), subtotal filling (Grade B), entry remnant (Grade C), or no filling (Grade D).

\section{MRI Findings}

A prospectively established protocol was used, including brain MRI with 3D DWI, FLAIR, and T2* sequences, and was performed 24 hours before embolization, 24-48 hours after the procedure was terminated, and at the 3-month follow-up. A comparison of the MR images obtained before treatment, 24-48 hours after treatment, and at the 3-month follow-up was performed and findings were recorded in an electronic database as quantitative data.

Magnetic resonance imaging was performed using a 3-T system (Achieva, Philips Medical Systems). DWI comprised a multisection, single-shot, spin-echo echo-planar imaging sequence. Diffusion gradients were applied in each of the $x, y$, and $z$ directions with two $b$ values $(0$ and $1000 \mathrm{sec} / \mathrm{mm}^{2}$ ). Imaging parameters included a field of view of $24 \mathrm{~cm}$, a $116 \times 97$ matrix, a TE of $69 \mathrm{msec}$, a section thickness of $5 \mathrm{~mm}$, and an intersection gap of $1 \mathrm{~mm}$. T2-weighted spin-echo imaging with a FLAIR sequence was also performed at each examination.

\section{Imaging Data Organization}

Abnormalities detected on DWI were recorded with regard to their number, location, and size. A neuroradiology brain anatomical atlas ${ }^{20}$ of intracranial arterial territories was used as the initial reference, but correlations with DSA were also used. Special attention was paid to the abnormality's territorial relationship with the arteries covered by the stent as well as the arteries harboring the aneurysm and the stent(s). Large territorial lesions were defined as lesions with a diameter greater than $20 \mathrm{~mm}$ on DWI.

Findings noted on postprocedural MRI and 3-month follow-up DWI were classified according to the relationship with the arterial branches related to the aneurysm treatment as follows: new (nonexistent on previous MRI) DWI hyperintensities distal to the arterial branch covered by the flow-diverting stent, DWI hyperintensities distal to the parent artery of the aneurysm (the stented artery), DWI hyperintensities distal to the same region of perfusion but not involving the 2 arterial branches mentioned above, and DWI hyperintensities distal to a region of perfusion not involving the stent-treated artery.

Control DSA findings at 3 months were additionally classified as in-stent stenosis or stent occlusion for the stented arterial segment and as side-branch remodeling/ 
narrowing or occlusion for the arterial branches covered by flow diverters at their ostia.

\section{Statistical Analysis}

Descriptive statistics were applied to the data, after verifying normality using the D'Agostino-Pearson test for normal distribution. The Fisher exact test was used for qualitative data and the chi-square test and the MannWhitney U-test (2-tailed) for quantitative data. Patient groups were compared using univariate ANOVA.

\section{Group Comparisons}

For purposes of comparison, we categorized aneurysms located at distal intracranial arterial bifurcations at or above the level of the circle of Willis (ACoA, ACA, MCA, and carotid T) as "supra-Willis" in location and aneurysms located at intracranial arterial bifurcations at and below the circle of Willis (carotido-ophthalmic, cICA, PCoA, AChA, and VA) as "infra-Willis" in location. This classification was chosen based on the location of the aneurysms, as well as on the dimensions of the stented artery. In the supra-Willis group the stented arteries are small-caliber arteries like the ACA and MCA, while the infra-Willis group includes larger-diameter stented arteries, such as ICA segments and the VA. We compared intraprocedural, postprocedural, and clinical complications developing in aneurysms in these 2 groups.

\section{Supplementary Comparison With the Department's Series of Cases of Stent-Assisted Coiling for the Same Time Period}

We statistically compared (1-way ANOVA) the number of DWI hyperintensities postoperatively and at follow-up. We also compared the patients with DWI-detected lesions in the study group with those treated by stent-assisted coiling for intracranial aneurysms during the same time period. The 2 groups had been previously compared statistically regarding age, sex, and treatment etiology to ensure statistical similarity in baseline characteristics between the groups. A Student t-test was used to test distributions of continuous variables. The chi-square test was used to analyze binary variables; for ordinal variables, the Wilcoxon rank-sum test was used to test the distribution between the groups.

Statistical analyses were performed using appropriate statistical electronic software (MedCalc Software) and the level of statistical significance was determined as $p \leq 0.05$.

\section{Results \\ Patient Population}

Over the course of an 18-month period, 49 intracranial aneurysms in 38 consecutive patients ( 7 males and 31 females) with a mean age of $53 \pm 13.5$ years $( \pm \mathrm{SD})$ were treated using flow-diverting stents. Five patients had been excluded from the study because they had undergone treatment of multiple aneurysms not only with flow diverters but also with other conventional techniques during the same session. Reasons for treatment included recurrence of previously embolized aneurysms in 17 patients (44.7\%), aneurysm rupture in 1 patient (2.6\%), aneurysm incidentally found due to an embolic complication in 2 patients (5.4\%), and unruptured aneurysms in 18 patients (47.3\%) discovered because of visual loss in 2 patients, hypopituitarism due to pituitary stalk compression in 1 patient, and headaches and atypical symptoms in the remaining patients.

\section{Aneurysm Characteristics}

Forty-nine aneurysms were treated; 39 were saccular (79.6\%), 9 were blood blister-like (18.4\%), and 1 was fusiform (2.0\%). Five aneurysms were located in the ACoA $(10.2 \%), 4$ in the ACA (8.2\%), 12 in the MCA (24.5\%), 1 at the AChA (2.0\%), 15 in the carotido-ophthalmic region $(30.6 \%), 3$ at the PCoA (6.2\%), 5 at the intradural cICA $(10.2 \%), 3$ at the carotid $\mathrm{T}$ junction (6.1\%), and 1 at the $\mathrm{V}_{4}$ segment of the VA (2.0\%).

Twenty-six aneurysms were small (diameter $<7 \mathrm{~mm}$ ), 5 were medium $(7-12 \mathrm{~mm}), 8$ were large $(12-24 \mathrm{~mm})$, and 2 were giant $(>24 \mathrm{~mm})$. The remaining aneurysms were very small blood blister-like aneurysms that could not be evaluated due to their size. Seventy-six percent $(n=37)$ of the aneurysms were wide necked. The mean dome-toneck ratio was $1.5 \pm 0.7 \mathrm{~mm}( \pm \mathrm{SD})$ and the mean aspect ratio was $1.7 \pm 0.7 \mathrm{~mm}$.

Overall, $40(82 \%)$ of the aneurysms treated were anatomically challenging for conventional techniques.

\section{Treatment and Technical Outcomes}

Forty-nine aneurysms were treated using 50 flow-diverting stents. All aneurysms were successfully embolized (100\% technical success rate). Five patients (13\%) were treated simultaneously for at least 2 aneurysms located in the same arterial segment. Single flow-diverter coverage was performed for 37 aneurysms (75.5\%), double coverage for $10(20.4 \%)$, and triple coverage for $2(4.0 \%)$. In 43 aneurysms (87.8\%) no adjunctive material, other than a flow diverter, was used, while for 5 cases (10.2\%) coils were also used and in 1 case $(2.0 \%)$ Onyx was used as a supplement to coils, to ensure early total occlusion in a patient who was soon returning to his country.

Three patients exhibited clopidogrel resistance (PRU values before adjustment are given in Table 1), but after a triple dose of the drug was administered, the PRU values normalized and thus these patients were included in the study. Two nonobese and nondiabetic patients exhibited aspirin resistance (ARU values before adjustment are given in Table 1) and acquired normalized values after supplementary treatment ( $250 \mathrm{mg}$ aspirin by mouth morning and evening), and thus were also included in the study.

Technical difficulties were encountered in 5 patients. Adjunctive balloon dilation using a HyperGlide balloon system (Covidien/ev3 Inc.) was needed for proper apposition of the stent against the aneurysm wall in 2 cases (Cases 25 and 26 in Table 1).

\section{Immediate and Follow-Up Clinical Outcomes}

The initial mRS scores were 0,1 , and 3 in 33 patients (86.8\%), 4 patients (10.5\%), and 1 patient (2.6\%), respectively (Table 1). The clinical situation postembolization 


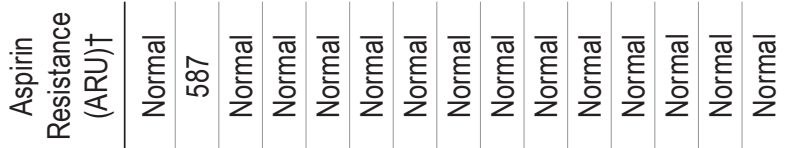

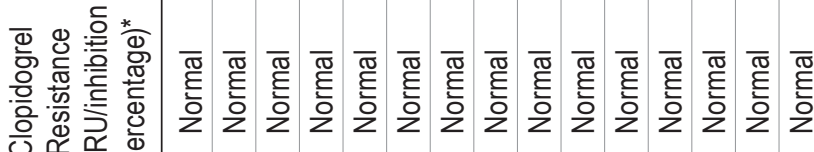
迹这

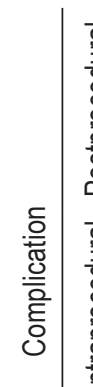
毫

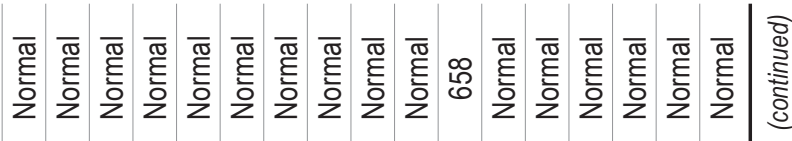

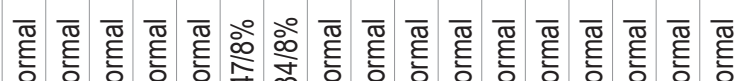

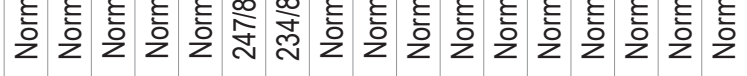

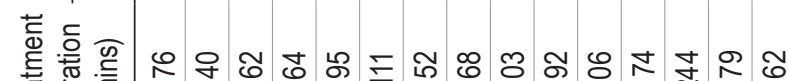
奠产

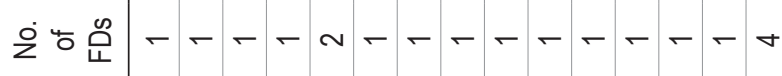

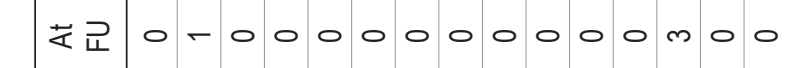

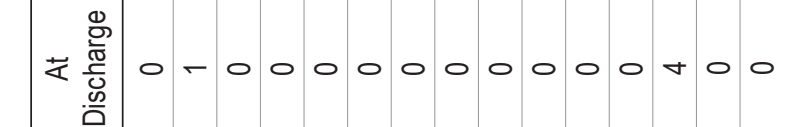
息 状 索峦 $0-00-00000001000$

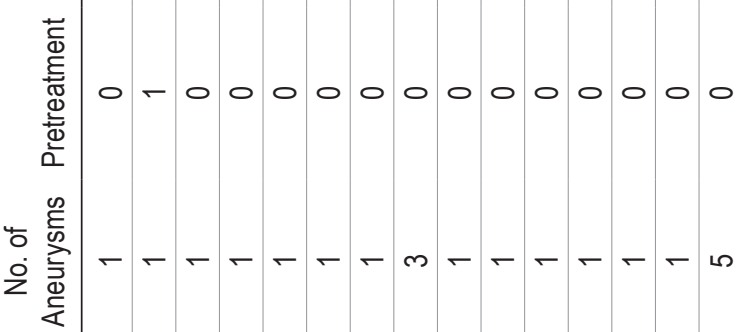

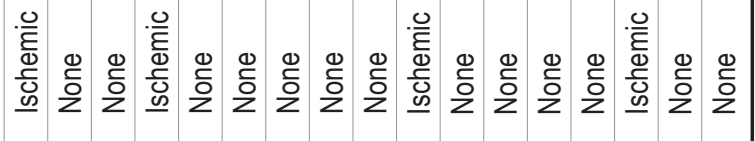
Ф)

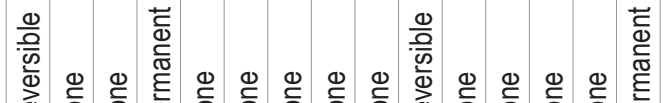

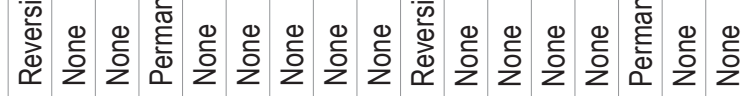

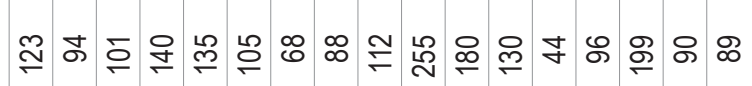

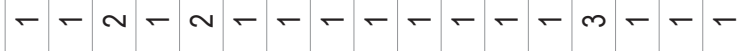
$000 n 00000-0000 m-0$ $000 n 00000-00004 n 0$

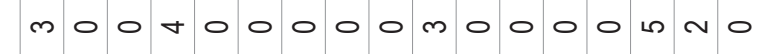




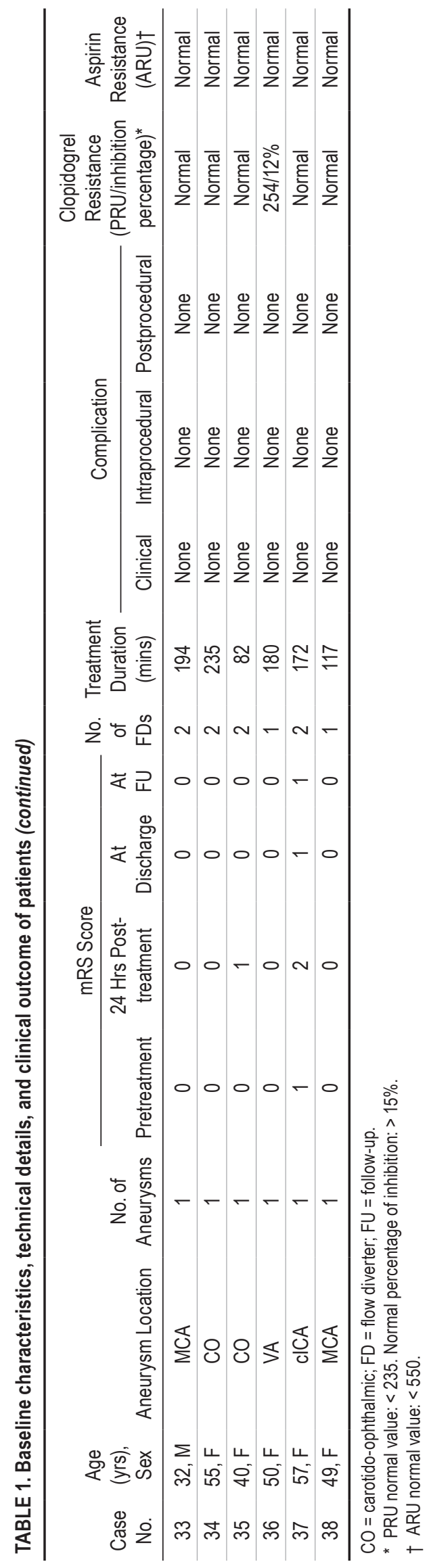

was unchanged for 30 patients (78.9\%), a delay in awakening occurred in 1 patient (2.6\%), and clinical deterioration compared with the preembolization state was found in 7 patients (18.4\%). The mRS scores at discharge were $0-1$, 2 , and 4 for 34 patients (89.4\%), 2 patients (5.3\%), and 2 patients (5.3\%), respectively. The follow-up mRS scores were $0-1,2$, and 3 for 35 patients $(92.1 \%), 1$ patient $(2.6 \%)$, and 2 patients $(5.3 \%)$, respectively.

\section{Complications and Clinical Impact}

Three intraprocedural thrombus formations $(7.8 \%)$ occurred. Five procedure-related, FLAIR-confirmed, ischemic events $(13.2 \%)$ occurred. No delayed rupture, stent migration, or mass effects (perianeurysmal brain inflammatory reaction) were found during hospitalization and follow-up.

Overall, 33 patients $(86.8 \%)$ were free of clinical complications during the first 3 postprocedural months, while $5(13.1 \%)$ experienced new neurological symptoms in the postoperative period. In 3 patients (7.8\%) the deficit was permanent at follow-up. Regarding the 35 patients who were free of new symptoms at the 3-month follow-up, there was no difference in cognitive test results between pretreatment and 3-month assessments. For the remaining 3 patients, there was no statistically significant change in cognitive test results before and after treatment.

\section{DSA Outcomes}

\section{Immediate and Follow-Up DSA}

Digital subtraction angiography was performed to determine the recanalization status of aneurysms before any retreatment was performed. At that time, $82.3 \%$ of the aneurysms had recanalized and $17.7 \%$ had some residual neck filling. Immediately after the procedure, there were 26 OKM Grade A aneurysms (53.0\%), 10 Grade B aneurysms (20.0\%), 7 Grade C aneurysms (14.3\%), and 6 Grade $\mathrm{D}$ aneurysms $(12.2 \%)$. At a mean of $187 \pm 51$ days of follow-up, there were 40 OKM Grade D aneurysms (81.6\%) and 9 Grade $\mathrm{C}$ aneurysms (18.4\%).

\section{Parent Artery and Collateral Branches on Follow-Up DSA}

Side-branch occlusions were evident in 8 patients $(21 \%$ of the patients, $16.3 \%$ of the treated aneurysms). There were no late DWI manifestations or clinical impact in any of these cases (mRS Score 0 at 3 months for all). The side branches concerned were ACoA segments and MCA branches in 3 cases, carotido-ophthalmic in 6 cases, and PCoA in 1 case.

Side-branch narrowing/remodeling was found in 7 cases (18.4\% of patients, $14.3 \%$ of treated aneurysms) and consisted of 6 cases of carotido-ophthalmic narrowing without any clinical impact and 1 case of a remodeled MCA branch, in which a clinically silent focal lesion was detected on DWI (follow-up mRS Score 0) (Fig. 1). Intrastent occlusion with subsequent side-branch thrombosis occurred in 1 patient (Case 13) who underwent retreatment of a previously ruptured MCA aneurysm. This patient had an mRS score of 3 at follow-up.

Overall, 15 side-branch modifications (30.6\%) were evident at follow-up, with no clinical impact on neurological 

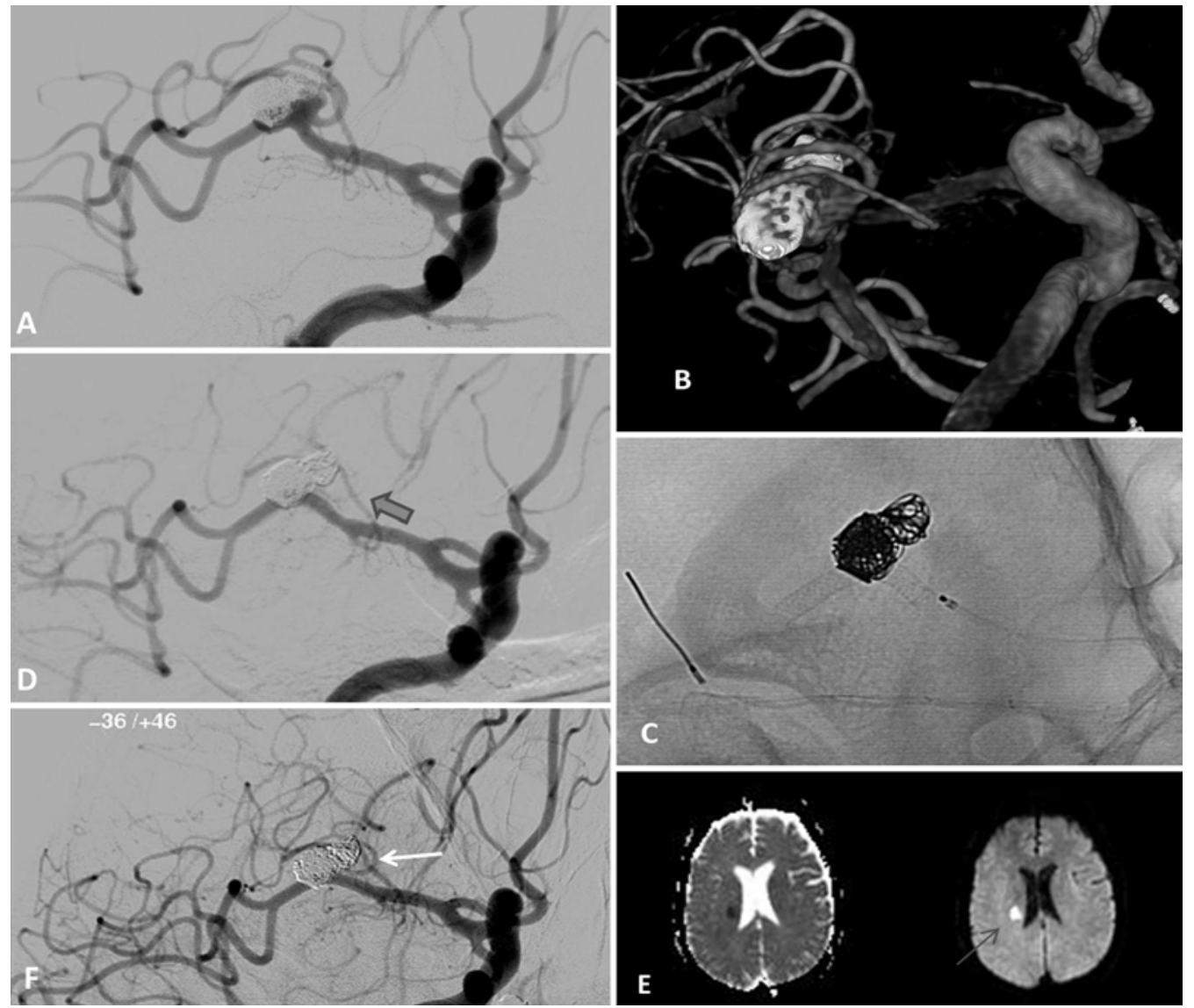

FIG. 1. Images obtained in a 58-year-old patient who underwent treatment for recanalization of a previously coiled right MCA aneurysm. A: Right ICA injection angiogram, working projection, obtained before treatment, showing residual aneurysm and MCA bifurcation branches emerging from the aneurysm's neck. B: 3D rotational angiography with double volumetric reconstruction. The coil mesh is evident. C: The flow-diverting stent is fully deployed, with the delivery wire still in place. D: Immediate angiographic outcome after flow-diverting stent placement; note the flow diversion effect on the covered side branch (thick arrow), which is nearly invisible after contrast medium injection. E: Diffusion-weighted image (right) and apparent diffusion coefficient map (left) obtained within the first 24 postoperative hours, showing a right parietal watershed region of diffusion restriction (arrow) that was clinically silent. F: Six-month follow-up angiogram, working projection, showing complete exclusion of the aneurysm. Note the remodeling (narrowing) of the side branch (arrow), compared to what was seen on the preoperative working projection image, as well as its reappearance, compared to the immediate post-procedure control. The latter is attributed to the continuing aspiration effect (reflected by the silent ischemic DWI lesion), which sustains adequate pressure gradient between the ostium and the distal brain parenchyma, allowing partial restoration of blood flow inside the covered branch. The patient's follow-up mRS score was 0 .

or cognitive status of the patients and with only 1 DWIdetected finding.

\section{MRI Outcomes}

\section{Immediate MRI Outcomes by Branch Territory}

At least 1 new hyperintense lesion was evident on postprocedural DWI in 35 patients $(92.1 \%)$. In 31 patients (81.6\%) 1-3 hyperintense lesions were found on DWI performed within 48 hours (mean $13.4 \pm 9.7$ hours) after the procedure (Fig. 2). Ten patients $(26.3 \%$ ) had 1 DWIdetected lesion, 12 patients $(31.6 \%)$ had 2 lesions, 9 patients $(23.7 \%)$ had 3 lesions, 1 patient $(2.6 \%)$ had 4 lesions, 2 patients $(5.3 \%)$ had 6 lesions, and 1 patient (2.6\%) had 7 lesions, with all territories considered.

In 3 patients (7.8\%) the lesions were clinically evident as permanent deficits and in $2(5.3 \%)$ as transient neurological deficits. In the remaining 33 patients $(86.9 \%)$, the le- sions remained clinically silent during the entire 3-month postprocedural period $\left(\chi^{2}=22.694, \mathrm{df}=12, \mathrm{p}=0.0304\right)$.

Concerning DWI-detected lesions distal to the side arterial branches (branches covered by flow diverters at their ostia), there were no new findings for $38(77.6 \%)$ of the treated aneurysms, 1 new lesion in 8 cases $(16.3 \%)$, and 2, 4 , and 7 new lesions in 1 case each (2.0\% for each case). Regarding DWI-detected lesions distal to the stented arterial segment (the segment harboring the aneurysm), there were no new lesions in 31 cases (63.3\%); 1 new lesion in 11 cases (22.4\%); 4 and 6 new lesions in 2 cases each (4.1\%); and 2,3 , and 9 new lesions in 1 case each $(2.0 \%)$.

As far as the same region of perfusion, but not the parent artery, there were no DWI-detected lesions in 41 cases (83.7\%), 1 and 2 new lesions in 3 cases each (6.1\%), and 3 new lesions in 2 cases $(4.1 \%)$. In the other regions of perfusion, not involved by the embolization, only 1 DWIdetected lesion was found in 1 patient. There was no statis- 


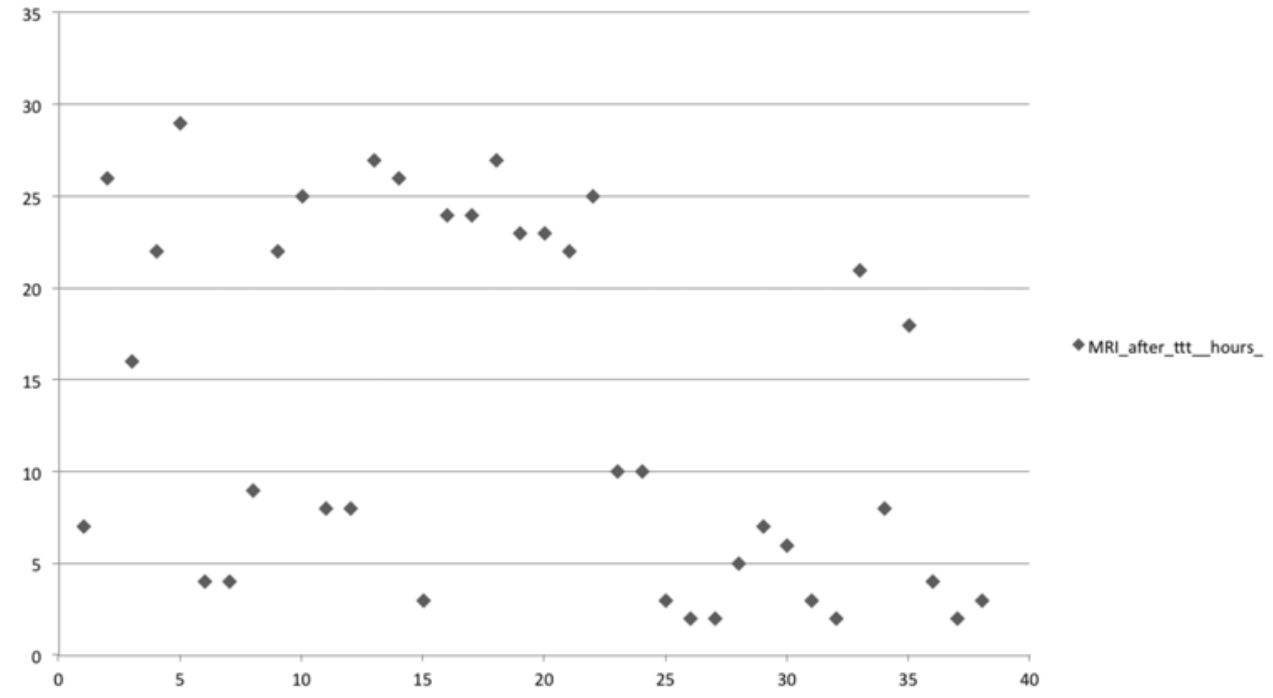

FIG. 2. Graph showing the time points at which each patient underwent postoperative MRI after completion of procedure. The values on the $x$ axis indicate the case number and those on the $y$ axis indicate the time from treatment to postoperative MRI in hours.

tically significant difference between the number of DWIdetected lesions distal to the covered side branches versus those distal to the stented arterial segment.

\section{Follow-Up MRI Comparison by Branch Territory}

Three late-occurring DWI-detected lesions (6.1\%) were documented in watershed locations; none of these lesions had been detected on postprocedural MRI. The covered branch was an MCA bifurcation in all cases, and all 3 patients (Cases 6, 10, and 24) had an mRS score of 0 at 3 months. None of these patients experienced intraprocedural or postprocedural complications, and only 1 of these patients harbored a lesion that was evident on FLAIR after a few days; this lesion was still clinically silent.

Compared with the postprocedural MRI, the 3-month follow-up DWI did not detect any new lesions distal to the stented parent artery in the same region of perfusion or in any other (not relevant to the procedure).

\section{Comparison of Distal Versus Proximal Intracranial Arterial Bifurcations}

There were 24 aneurysms in the supra-Willis group (49\%) and 25 aneurysms in the infra-Willis group (51\%). Two intraprocedural complications occurred, one in a supra-Willis aneurysm and the other in an infra-Willis aneurysm; no statistically significant difference could be established $\left(\chi^{2}=0.001\right.$, df $\left.=1, p=0.9709\right)$. Three postprocedural complications occurred in the supra-Willis group and 2 occurred in the infra-Willis group without a statistically significant result ( $\chi^{2}=0.002, \mathrm{df}=1, \mathrm{p}=0.9616$ ). Overall, there was no statistically significant difference between the 2 groups in terms of clinical complications $\left(\chi^{2}=2.867, \mathrm{df}=2, \mathrm{p}=0.2385\right)$, and there was no statistically significant difference in mRS scores at the 3-month follow-up.

\section{Multifactorial Analysis of DWI-Detected Findings}

Multiple regression analysis (with a stepwise method) of covered side-branch DWI-detected lesions-early and late appearing - using different dependent variables (number of flow diverters, intraprocedural complications, and territory of the treated aneurysm) did not show any statistically significant correlation. In contrast, regarding postprocedural DWI-detected lesions in the territory of the parent (aneurysm-hosting) artery, in the multiple regression analysis the number of flow diverters used was the only statistically significant factor (F-ratio $=4.9981$, $\mathrm{p}=0.030)$.

\section{Supplementary Comparison With the Department's Series of Stent-Assisted Coiling Procedures During the Same Time Period}

During the same time period, 42 patients ( 9 males and 33 females) harboring 42 aneurysms underwent stentassisted intracranial aneurysm coiling. The mean age of these patients was $56.6 \pm 11.3$ years. Ten patients $(23.8 \%)$ underwent treatment for recanalization, 1 patient $(2.4 \%)$ for $\mathrm{SAH}$, and 31 patients (73.8\%) for unruptured aneurysms. The preprocedure $\mathrm{mRS}$ scores were $0,1,2$, and 3 for 36 patients (87.8\%), 3 patients (7.3\%), 1 patient $(2.4 \%)$, and 1 patient (2.4\%), respectively. At discharge mRS scores were $0,1,2$, and 3 for 35 patients (83.3\%), 2 patients (4.8\%), 3 patients (7.1\%), and 2 patients (4.8\%), respectively.

The 3-month follow-up mRS scores were $0,1,2$, and 3 for 35 patients (83.3\%), 3 patients (7.1\%), 3 patients (7.1\%), and 1 patient (2.4\%), respectively. Intraprocedural complications included 1 aneurysm rupture (2.4\%) and 2 thrombus formations (4.8\%), which both resolved after injection of Reopro in situ during the procedure. In 1 case the stent was used as a bailout technique for a protrusion of coil during treatment of a ruptured MCA aneurysm. With regard to baseline characteristics, this group did not statistically significantly differ from the flow diverter-treated group.

A total of 68 DWI-detected silent lesions were found in 15 of these patients (35.7\%), while for the remaining $61.9 \%$ of the cases there were no DWI-detected silent lesions. Absence of delayed DWI-detected findings was confirmed on follow-up MRI. One-way ANOVA regarding the num- 
ber of DWI-detected lesions postoperation between the 2 groups (flow diverter vs stent-assisted coiling) revealed an F-ratio of 4.354, with a significance level of $\mathrm{p}=0.040$.

There were no statistically significant differences regarding follow-up mRS scores and complication rates between the 2 groups.

\section{Discussion}

Flow-diverting stents offer new options for the treatment of intracranial aneurysms, increasing the number of aneurysms that can be treated endovascularly and allowing for improved long-term occlusion rates., ${ }^{3,13,14,19}$ In a study by Brinjikji et al., $54 \%$ of aneurysms in 200 patients were deemed likely to be amenable to treatment using flow diverters, the difficulty being to select patients for whom treatment is necessary and justified regarding therapeutic alternatives and their associated benefits and risks. As reported in recent meta-analyses, early and late complication rates of the technique were not negligible., ${ }^{2,6}$

At present, inhomogeneity of practices exists regarding intracranial arterial locations where flow diverters are considered safe for use. In the US flow diverters are FDA approved only for ophthalmic segments of the ICA. In Europe and outside the US, however, an increasing number of cases are being reported in which flow diverters have been used in other intracranial locations, even beyond the circle of Willis, ${ }^{17}$ pinpointing the potential of the device for these locations. At the same time challenges regarding safety in these locations remain and need to be addressed accordingly. In the meantime, the present study, although limited by its sample size and design, may provide some useful insight into the matter, reflecting everyday clinical challenges and subjects for further research.

Ischemic micro-lesions related to the procedure occur far more often than anticipated, especially clinically silent lesions, which occurred in $33(86.8 \%)$ of the patients in our series, most often presenting with few and focal DWI spots. No statistical difference in early and late clinical or DWI-correlated complication rates could be established between supra-Willis or infra-Willis stented aneurysm locations, suggesting that both locations benefit from the device without a statistically important difference in complication rates.

Early-occurring DWI-detected lesions are most prominent distal to the treated region of perfusion, suggesting that silent micro-ischemic events occur as a consequence of the procedure. This is in accordance with findings from previous studies conducted in patients who underwent endovascular coiling, either simple or assisted., ${ }^{1,4}$ Nevertheless, the number of lesions is significantly higher when flow-diverting stents are used $(86 \%$ vs $20 \%-61 \%$ published for simple or assisted coiling), even compared with stent-assisted coiling (64\% silent ischemic events in published series, ${ }^{10} 35.7 \%$ in our patients who underwent stent-assisted coiling, with a statistically significant difference; $p=0.04$ ), revealing an increased embolic tendency with this type of device, probably due to its mechanical properties and to the technical manipulations during the procedures. Endovascular procedures, regardless of their nature, seem to have a tendency to result in microembolic events, the vast majority of which remain silent when ap- propriate anti-aggregation treatment and technique rules are respected, and do not seem to hinder the safety of the procedure.

In the multivariate analysis, there was no statistically significant difference regarding aneurysm territory, number of flow diverters used, or complication rate; however, the limited sample is one of the main limitations in our study. The fact that the number of flow diverters used was the only statistically significant factor regarding ischemic complications simply reflects the risk yielded by complex endovascular procedures.

Regarding delayed DWI-detected lesions in the present study, only territories of side branches were involved. These are presumably associated with hemodynamic and not embolic factors. Recent studies, both in animals ${ }^{11,12}$ and in clinical practice, ${ }^{19}$ suggest that in the case of laminar flow, due to a high pressure gradient in the covered side branch, the side branch remains patent, even with multiple overlapping devices. ${ }^{8}$ Thus, in cases in which an important collateral network exists or develops, a "competition" of flow may have as a result a decrease in pressure gradient between the covered ostium and the distal portion of the arterial branch, contributing in such a way to a hemodynamic "stagnation."

Leptomeningeal collaterals are small arterial anastomoses situated distally at the level of terminal cortical branches, along the surface of the brain. This collateral supply system is more or less underused under normal conditions, but is recruited when a major artery is either chronically or acutely occluded. ${ }^{7}$ The fact that all 3 cases of delayed DWI-detected spots in our series occurred in MCA branches, where partial compensation of flow is provided by leptomeningeal anastomoses, sustains this hypothesis.

The limited areas of infarction, with absent or mild and transient clinical symptoms, suggest the presence of a flow competition by the collateral arterial network, which could cause a progressive deconstruction of the stent-covered branch, with no or mild symptoms of transient ischemia during the process of collateral network development. Because the symptoms are absent or mild and transient, this phenomenon is largely underestimated in clinical series. The fact that almost half of the patients (49.5\%) had asymptomatic remodeling (narrowing or occlusion) of covered side branches further supports this hypothesis. While the collateral network is progressively developed in the months after embolization, the covered arterial branches experience a pressure gradient decrease, which allows further endothelialization of the covered ostia and narrowing of the underused arterial branch. This pathophysiological concept is interesting in terms of therapeutic strategy, as a potential factor to consider for limiting the risk of sidebranch thrombosis.

Similarly, in the cases of coverage of the ophthalmic artery ostium, collateral supply by branches of the ECA provide a "competition" of flow for the supply of the retina, resulting again in a pressure gradient drop along the ophthalmic artery, evident at follow-up as an occlusion or nonvisualization of the latter by the selective ICA angiogram, with intact visual function, as the central retinal artery is still supplied by the ECA anastomotic network. 
While early side-branch occlusions occur mainly as a result of in-stent thrombosis and procedure-related complications in general, delayed occlusions or remodeling remain clinically silent, even though the occasional presence of focal DWI-detected spots reflects the hemodynamic stress under which a collateral network develops and gradually takes over part of the territory of the side branch.

\section{Limitations}

This study reflects a single-center experience; consequently, the sample size and the number of findings in the different subgroups limit the power of the study. Nevertheless, our findings may be indicative of certain neurovascular mechanisms and considerations that may be worthy of further elucidation with appropriately designed research.

\section{Conclusions}

Silent DWI-detected lesions related to the procedure occur far more often than anticipated, but the technique of treating aneurysms using flow-diverting stents remains safe and effective, providing an interesting alternative for "difficult" aneurysms, regardless of supra- or infra-Willis aneurysm localization.

Nevertheless, in dealing with branches that are bound to be covered by flow diverters, which is often the case in supra-Willis localizations, meticulous case-by-case study of the collateral supply to the vascular territory in question is more than necessary, especially with regard to anastomotic supply, since branches that do not receive direct collateral supply from the circle of Willis (MCA branches) are in danger of late-occurring hemodynamic-related micro-ischemic lesions.

Overall, with careful planning, flow diverter treatment for intracranial aneurysms remains a safe and effective technique.

\section{References}

1. Albayram S, Selcuk H, Kara B, Bozdag E, Uzma O, Kocer N, et al: Thromboembolic events associated with balloon-assisted coil embolization: evaluation with diffusion-weighted MR imaging. AJNR Am J Neuroradiol 25:1768-1777, 2004

2. Arrese I, Sarabia R, Pintado R, Delgado-Rodriguez M: Flow-diverter devices for intracranial aneurysms: systematic review and meta-analysis. Neurosurgery 73:193-200, 2013

3. Becske T, Kallmes DF, Saatci I, McDougall CG, Szikora I, Lanzino G, et al: Pipeline for uncoilable or failed aneurysms: results from a multicenter clinical trial. Radiology 267:858868, 2013

4. Biondi A, Oppenheim C, Vivas E, Casasco A, Lalam T, Sourour N, et al: Cerebral aneurysms treated by Guglielmi detachable coils: evaluation with diffusion-weighted MR imaging. AJNR Am J Neuroradiol 21:957-963, 2000

5. Brinjikji W, Cloft HJ, Fiorella D, Lanzino G, Kallmes DF: Estimating the proportion of intracranial aneurysms likely to be amenable to treatment with the pipeline embolization device. J Neurointerv Surg 5:45-48, 2013

6. Brinjikji W, Murad MH, Lanzino G, Cloft HJ, Kallmes DF: Endovascular treatment of intracranial aneurysms with flow diverters: a meta-analysis. Stroke 44:442-447, 2013

7. Brozici M, van der Zwan A, Hillen B: Anatomy and functionality of leptomeningeal anastomoses: a review. Stroke 34:2750-2762, 2003

8. Dai D, Ding YH, Kadirvel R, Rad AE, Lewis DA, Kallmes
DF: Patency of branches after coverage with multiple telescoping flow-diverter devices: an in vivo study in rabbits. AJNR Am J Neuroradiol 33:171-174, 2012

9. Fiorella D, Lylyk P, Szikora I, Kelly ME, Albuquerque FC, McDougall CG, et al: Curative cerebrovascular reconstruction with the Pipeline embolization device: the emergence of definitive endovascular therapy for intracranial aneurysms. J Neurointerv Surg 1:56-65, 2009

10. Hahnemann ML, Ringelstein A, Sandalcioglu IE, Goericke S, Moenninghoff C, Wanke I, et al: Silent embolism after stent-assisted coiling of cerebral aneurysms: diffusionweighted MRI study of 75 cases. J Neurointerv Surg 6:461465, 2014

11. Kallmes DF, Ding YH, Dai D, Kadirvel R, Lewis DA, Cloft HJ: A new endoluminal, flow-disrupting device for treatment of saccular aneurysms. Stroke 38:2346-2352, 2007

12. Kallmes DF, Ding YH, Dai D, Kadirvel R, Lewis DA, Cloft HJ: A second-generation, endoluminal, flow-disrupting device for treatment of saccular aneurysms. AJNR Am J Neuroradiol 30:1153-1158, 2009

13. Lubicz B, Collignon L, Raphaeli G, De Witte O: Pipeline flow-diverter stent for endovascular treatment of intracranial aneurysms: preliminary experience in 20 patients with 27 aneurysms. World Neurosurg 76:114-119, 2011

14. Lylyk P, Miranda C, Ceratto R, Ferrario A, Scrivano E, Luna $\mathrm{HR}$, et al: Curative endovascular reconstruction of cerebral aneurysms with the pipeline embolization device: the Buenos Aires experience. Neurosurgery 64:632-633, 2009

15. Nelson PK, Lylyk P, Szikora I, Wetzel SG, Wanke I, Fiorella D: The pipeline embolization device for the intracranial treatment of aneurysms trial. AJNR Am J Neuroradiol 32:34-40, 2011

16. O'Kelly CJ, Krings T, Fiorella D, Marotta TR: A novel grading scale for the angiographic assessment of intracranial aneurysms treated using flow diverting stents. Interv Neuroradiol 16:133-137, 2010

17. Pistocchi S, Blanc R, Bartolini B, Piotin M: Flow diverters at and beyond the level of the circle of willis for the treatment of intracranial aneurysms. Stroke 43:1032-1038, 2012

18. Raymond J, Guilbert F, Weill A, Georganos SA, Juravsky L, Lambert A, et al: Long-term angiographic recurrences after selective endovascular treatment of aneurysms with detachable coils. Stroke 34:1398-1403, 2003

19. Szikora I, Berentei Z, Kulcsar Z, Marosfoi M, Vajda ZS, Lee $\mathrm{W}$, et al: Treatment of intracranial aneurysms by functional reconstruction of the parent artery: the Budapest experience with the pipeline embolization device. AJNR Am J Neuroradiol 31:1139-1147, 2010

20. Tatu L, Moulin T, Vuillier F, Bogousslavsky J: Arterial territories of the human brain. Front Neurol Neurosci 30:99110, 2012

\section{Author Contributions}

Conception and design: Iosif, Mounayer. Acquisition of data: Iosif, Camilleri, Boncoeur-Martel. Analysis and interpretation of data: Iosif, Camilleri, Boncoeur-Martel, Mounayer. Drafting the article: Iosif, Camilleri, Mounayer, Yardin. Critically revising the article: all authors. Reviewed submitted version of manuscript: all authors. Approved the final version of the manuscript on behalf of all authors: Iosif. Statistical analysis: Iosif. Study supervision: Mounayer.

\section{Correspondence}

Christina Iosif, Department of Interventional Neuroradiology, Dupuytren University Hospital, 2 Avenue Martin Luther King, 87042 Limoges, France. email: christina.iosif@gmail.com. 